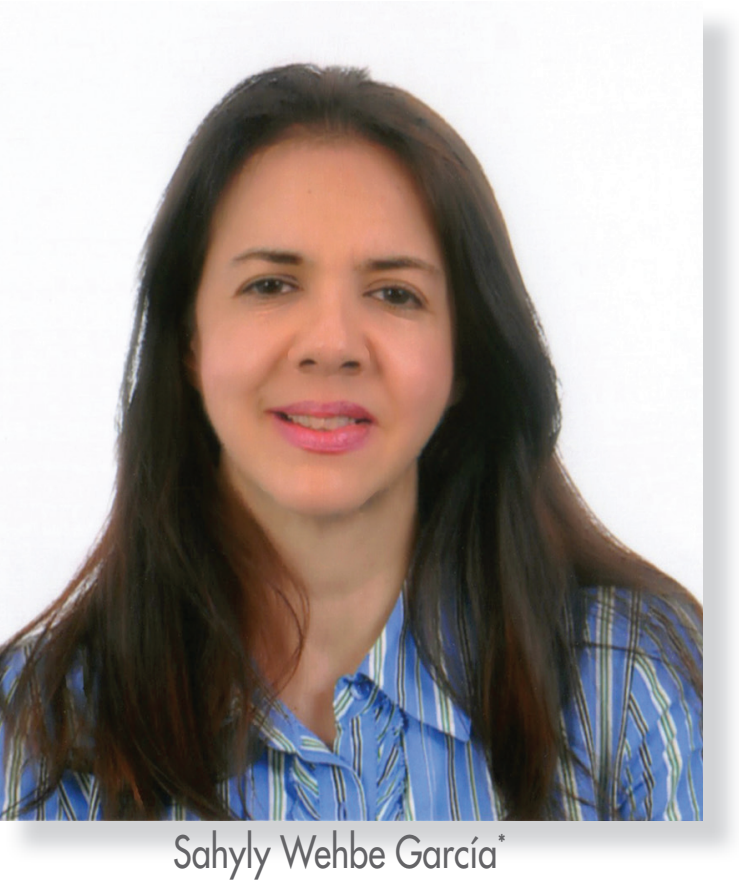

\title{
El resumen como estrategia para mejorar la comprensión lectora en la asignatura Derecho de los Contratos
}

\section{The summary strategy: Improving reading comprehension in law students}

Recibido: 27-01-16 Aprobado: 15-03-16

\section{Resumen}

Este proyecto de investigación se enfoca en la implementación de la estrategia del resumen para incrementar la comprensión lectora, luego de comprobarse la necesidad de que los estudiantes de Derecho se perfeccionasen en esa competencia, al haber constatado en el desarrollo de las clases que, cuando procedieron a resumir diversos textos, no evidenciaron una adecuada comprensión.

La estrategia se aplicó a 27 estudiantes, 5 hombres y 22 mujeres en el período académico de agosto a diciembre del 2014 en la Pontificia Universidad Católica Madre y Maestra, campus de Santiago, en un proceso de cuatro fases: la primera, diagnóstica; la segunda, socialización con intervención pedagógica; la tercera, prácticas de los estudiantes y en la cuarta fase se incluyó un texto para resumir en el examen final del grupo. Se usó una rúbrica para evaluar los resultados, constatando que fueron satisfactorios.

\section{Abstract}

This research project focuses on the implementation of the Summary Strategy as a means to increase reading comprehension levels in undergraduate Contract Law students.

The strategy was applied to 27 students, 5 men and 22 women, in the academic period between August and December 2014 at the Pontificia Universidad Catolica Madre y Maestra, Santiago campus. The implementation consisted of a four-step process: (1) diagnostic phase; (2) socialization with pedagogical interventions; (3) student practices; and (4) a summary as part of the students' final exam. The scoring guide used to evaluate student results indicates a positive relationship between the use of the Summary Strategy and students' reading comprehension levels.

\section{Palabras clave}

aprendizaje, resumen, comprensión lectora

\section{Keywords}

learning, summary, reading comprehension

*Sahyly Wehbe Garcia: Licenciada en Derecho por la Universidad Católica Madre y Maestra. Posee una especialidad en Docencia Universitaria y una Maestría en Ciencias Jurídicas por la Pontificia Universidad Católica Madre y Maestra. Es profesora a medio tiempo del Departamento de Ciencias Jurídicas, Facultad de Ciencias Sociales Y Administrativas, PUCMM. Para contactar a la autora: Sahyly.wehbe@yahoo.com 


\section{Introducción}

En la práctica universitaria resulta muy importante el desarrollo de competencias de lectura y escritura a través del currículo para que los estudiantes puedan empoderarse del saber disciplinar. En el caso específico de los estudiantes del área jurídica, es fundamental que comprendan de forma adecuada los textos con los cuales interactúan, debido a que constituyen una herramienta fundamental de trabajo. Sin embargo, frecuentemente esto no ocurre, pues muchos de ellos no logran construir un verdadero aprendizaje de lo estudiado, a partir de lo que comprenden. Frente a esta problemática, el rol del profesor resulta determinante en la tarea de colaborar con el desarrollo de la comprensión lectora mediante la implementación de estrategias adecuadas que lleven a los estudiantes a un mejor procesamiento de la información escrita.

Entre las diversas estrategias de estudio que permiten incrementar la comprensión lectora, se encuentra el resumen, pues a través de él se condensa y construye información a la vez. Tal como expresan Padilla, Douglas y López (2011), "Leer es una tarea compleja que no se limita al simple descifrado, sino que implica una actividad de gran coste cognitivo en la que interactúa la información que tenemos almacenada en la memoria con la que nos provee el texto" (p.73).

Con el interés de profundizar la comprensión de los contenidos de los textos en los estudiantes de Derecho, se trabajó la estrategia del resumen entre los estudiantes de la asignatura Derecho de los Contratos, grupo 001, en el período académico 1-2014-2015, en el marco del Diplomado en Lectura y Escritura a través del Currículo en el Nivel Superior impartido por el Centro de Excelencia para la Investigación y Difusión de la Lectura y Escritura (CEDILE) de la PUCMM, desde mayo a diciembre de 2014.

En el presente trabajo nos proponemos explicar las cuatro fases del proyecto de investigación-acción que llevamos a cabo, en las que la intervención pedagógica jugó un rol importante para apoyar el desarrollo de la jerarquización de la información escrita de parte de los estudiantes. Pretendemos demostrar que incluir el resumen como estrategia para mejorar la comprensión lectora en los estudiantes de Derecho de los Contratos, proporcionándoles herramientas para discriminar las ideas relevantes de las que no lo son, a la vez que a ayudándolos a reconocer los pasos específicos que se requieren para resumir textos, consiguieron mejorar sus competencias lectoras durante el transcurso del semestre.

\section{Desarrollo}

\section{Contextualización}

La asignatura Derecho de los Contratos es una materia obligatoria para los estudiantes de la carrera de Derecho de la Pontificia Universidad Católica Madre y Maestra. El derecho sobre contratos forma parte del área de las obligaciones, ubicadas en el Derecho Civil, que es la rama del Derecho que estudia y reglamenta las relaciones particulares de las personas, sus compromisos, su estado y relaciones jurídicas privadas (Romero Butten, p.15 2001).

Esta asignatura presenta un contenido amplio y variado, pues se compone de diez contratos diferentes con sus propias particularidades. Estos son: la compraventa, la permuta, el arrendamiento, el contrato de empresa, el mandato, el préstamo, el depósito, los contratos aleatorios, el contrato de seguro terrestre y la transacción. Los referidos contratos tienen aspectos comunes, pues todos son acuerdos obligatorios entre partes que deben de reunir condiciones específicas para su validez: consentimiento de las partes intervinientes, capacidad para contratar, un objeto cierto y una causa lícita (Código Civil Dominicano, 2013); sin embargo, cada uno de estos contratos también posee reglas individuales.

Por lo indicado anteriormente, se requiere que los alumnos inscritos en esta asignatura estudien textos con contenidos generales y específicos a la vez para poder entender y aplicar los conocimientos de esta útil e indispensable materia sobre contratos. Esto requiere de estrategias que, como la del resumen, faciliten el procesamiento de la información escrita de dichos textos. En este sentido, esta asignatura resulta ideal para la aplicación de esas estrategias debido al volumen de información que los estudiantes deben manejar en la misma.

Este proyecto de investigación en la acción se aplicó al grupo que cursó Derecho de los Contratos, en horario de 3:00 p.m. a 5:00 p.m. los martes y 3:00 p.m. a 4:00 p.m. los jueves desde el mes de agosto al mes de diciembre de 2014, correspondiente al primer ciclo del periodo 2014-2015. El curso contó con la participación de 27 estudiantes dominicanos, 5 hombres y 22 mujeres, todos los cuales estaban aproximadamente a un año de graduarse de la carrera de Derecho.

\section{Principios teóricos}

En lo que se refiere a la alfabetización académica, ésta se define como el proceso de enseñanza que puede o no ponerse en marcha para favorecer el acceso 
de parte de los alumnos a las acciones que han de realizar los docentes con apoyo de la institución a fin de que los estudiantes universitarios aprendan a manejar diversas estrategias, entre las que se encuentran argumentar, buscar información y resumir (Carlino, 2013). De modo que saber resumir constituye un tema clave de la alfabetización académica.

El resumen, definido de forma concisa, es aquel tipo de discurso que otorga una variante personal de una macro-estructura general del discurso que se procede a resumir (Van Dijk, 1980). En el mismo orden de ideas de lo expresado anteriormente, Padilla, Douglas y López (2010, p.46), expresan que el resumen "implica la construcción de un nuevo texto, a partir de los datos del texto fuente, los cuales pueden ser reorganizados y jerarquizados de distintas maneras de acuerdo con el propósito lector y el tipo de texto en cuestión". O sea, que los resultados de un resumen dependen en gran medida de lo que conoce la persona que lo realiza y de su propósito.

Van Dijk (1980) ha establecido reglas que permiten llevar a cabo el proceso de resumir de forma idónea. Son las denominadas macrorreglas, las cuales constituyen operaciones intelectuales que realiza el lector con el fin de obtener las informaciones relevantes del texto. Las mismas reconstruyen formalmente las deducciones de la macroestructura del texto. Estas son: omitir y seleccionar, generalizar y construir. Este proceso implica excluir las informaciones no relevantes y repetitivas, escoger los datos que se consideren indispensables para la comprensión del contenido, generalizarlos y, a partir de estos pasos, elaborar un nuevo texto.

El resumen constituye una excelente estrategia para mejorar la comprensión lectora, pues luego de la identificación de las ideas principales y secundarias, de la omisión y selección de ideas a partir de lo que los estudiantes han comprendido, pueden construir un nuevo texto, fundado en el que usaron de base, sin desvirtuar la esencia de ese texto, pero incluyendo a la vez sus propios criterios.

\section{Principios metodológicos}

El proyecto de investigación en la acción que se realizó sobre el resumen como estrategia para optimizar la compresión lectora se puso en práctica desde el inicio al final del periodo académico de agosto a diciembre de 2014, siendo el profesor el mismo investigador.

Durante el proceso se trabajó con resúmenes de diferentes textos sobre el tema de los contratos. Esto así debido al contenido de la asignatura en la que se llevó a cabo el proyecto, que requiere la comprensión de diferentes tipos de contratos, tal como se expresó anteriormente. La estrategia de resumen se aplicó, pues, a textos que representan cada tipología de contrato requerido.

El proceso constó de cuatro fases. La primera fase se realizó con un propósito diagnóstico, en la cual se asignó la realización de un resumen a equipos formados por varios estudiantes para trabajar un mismo resumen, con la idea de potenciar el trabajo colaborativo.

La segunda fase consistió en una intervención pedagógica, en la que se proporcionó información a los alumnos sobre lo que significa resumir, se trabajaron en clase estrategias para seleccionar la idea principal de cada párrafo de un texto, de manera grupal e individual. Asimismo, se realizaron ejercicios para aprender a omitir lo que no es necesario y para jerarquizar la información. También se realizaron prácticas sobre el uso de los conectores para apoyar la progresión temática y se compartieron los criterios para evaluar los resúmenes.

En la tercera fase se trabajó con los grupos ya formados para realizar resúmenes diferentes, que fueron evaluados mediante una rúbrica (presentada en la fase anterior) y realimentados, y, en la última fase, se incluyó un escrito para ser resumido en el examen final que tuvo puntuación asignada.

Los diferentes grupos estuvieron compuestos por los mismos estudiantes durante todo el proceso del proyecto de investigación-acción, quienes realizaron resúmenes sobre el contrato, eligiendo diferentes tipos del mismo. Se omiten los nombres de los alumnos por razones éticas.

En cuanto a la evaluación de los resúmenes, la rúbrica estuvo organizada alrededor de los siguientes criterios: presentación de la idea principal y de las ideas que la apoyan, aplicación de las macrorreglas en la elaboración del resumen, claridad y comprensibilidad en las informaciones expresadas, precisión y propiedad léxica, aplicación de procedimientos de cohesión en la construcción de las ideas, registro y extensión adecuados, dominio de la normativa.

\section{Análisis de los resultados}

En la primera fase del proyecto se realizó el resumen diagnóstico en el curso dividido en cinco grupos y se produjeron igual número de resúmenes. A continuación, se transcribe un ejemplo del primer resumen para mostrar cómo se realizó el análisis del mismo con la finalidad de diagnosticar cuáles eran las habilidades de los estudiantes en esta competencia. Luego se presentan, en la Figura N. 1 los resultados de la evaluación diagnóstica de los cinco resúmenes. 


\section{Historia de las compraventas}

La compraventa tiene sus inicios en el derecho arcaico, culminando con el Justiniano y fue definido por las XII tablas como un negocio al contado; mientras que Goyo establecía que para transferir el dominio de las cosas emancípales era requerida la mancipación.

Compraventa primitiva: La cual se desarrolló en la monarquía y era confundida con la mancipación; La compraventa Arcaica: que fue desarrollada bajo dos formas: compraventa al contado y compraventa a crédito.

La evolución de la compraventa representa 5 etapas distintas:

El acto mancipatio

El acto mancipatio: ha reemplazado el lingote que se pesaba por ases que se cuentan.

El acto mancipatio/emptio se destina a transferir el precio a distancia contra cierto número de ases que se colocan sobre la balanza.

Las partes acuerdan la cosa y su precio.

Se extiende la compraventa a su concepto general.

A modo de conclusión, la compraventa ha evolucionado en todo el comercio, pero más complejo de cómo se manejaba antes, actualmente tiene muchas trabas en la recesión en la negociación a nivel mundial.

En el resumen anteriormente presentado, existe falta de cohesión entre las ideas y una reconstrucción muy literal del texto base. Se trata, más bien, de una labor de reproducción del texto original, lo que evidencia escasa comprensión. Los participantes utilizaron listas de ideas, sin suficientes conectores entre ellas. Este análisis demuestra la importancia de trabajar estrategias discursivas en la clase para lograr un mejor procesamiento de la información escrita.

\section{Figura I}

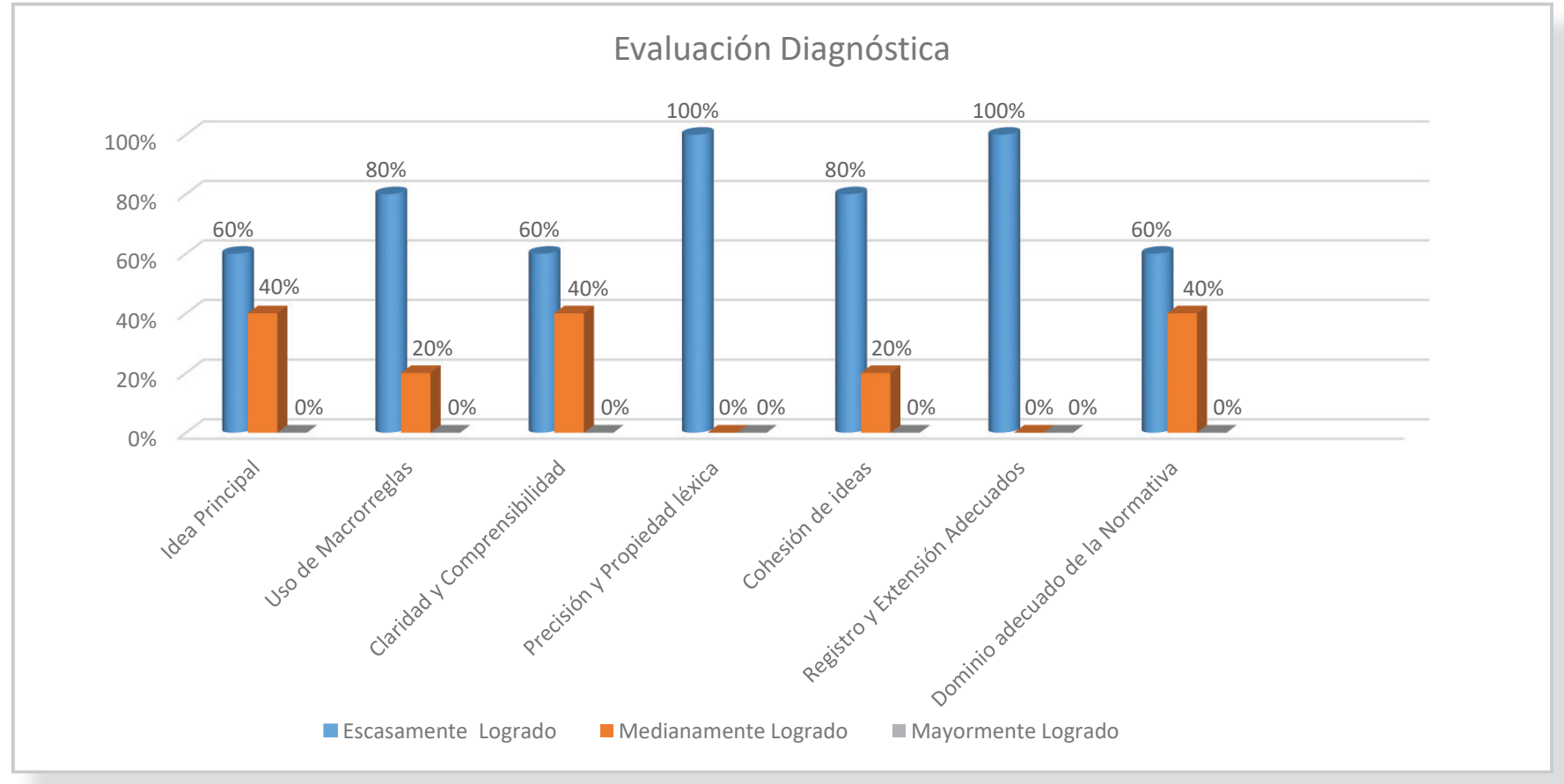

"El resumen como estrategia para la comprensión lectora aplicado a los estudiantes de la asignatura Derecho de los Contratos", Sahyly Wehbe Garcíal, Cuaderno de Pedagogía Universitaria Año 12/ N.24/ julio-diciembre 2015/ Santiago, República Dominicana/ PUCMM/ p. 44-51 


\section{$48 \cdot$ eCOS DESDE LAS FAcultades}

Se puede observar que, en los resúmenes de esta primera fase, los estudiantes mostraron dificultad para señalar cuáles eran las ideas principales, no aplicaron las macrorreglas en la elaboración del resumen, ni mostraron cohesión entre las ideas. Aunque fueron bastante claros en lo que deseaban expresar y mostraron una normativa aceptable, la extensión del resumen resultó muy larga. Además, utilizaron frecuentemente listas de ideas y presentaron una reconstrucción muy literal con respecto al texto base.

La segunda fase fue formativa, tal como se indicó anteriormente. Se llevó a cabo durante dos semanas. Se realizó una intervención pedagógica, dando explicaciones y asignando lecturas a los estudiantes, que se analizaron colectivamente para distinguir ideas principales de secundarias, como base para saber qué omitir al realizar un resumen; se escribieron ejemplos en la pizarra de los ejercicios realizados por los mismos estudiantes y realimentados por el profesor, mostrando cuáles ejemplos jerarquizaban mejor la información; también se trabajaron los diferentes tipos de conectores y se analizó cómo permitían relacionar las ideas de manera lógica lo que sentó las bases teóricas y prácticas para el trabajo que se realizaría posteriormente.

En la tercera fase, los estudiantes se dividieron en igual número de grupos, con los mismos miembros para hacer igual número de resúmenes. El trabajo colectivo ayudó a avanzar en el procesamiento de la información escrita, pues conllevó un mayor número de análisis y transformaciones del texto base que los que se ponen en marcha en situaciones de revisión individual e incidió en la regulación del proceso de escritura (Castelló, 2008).

A continuación, se muestra un ejemplo de un resumen trabajado en la tercera fase del proceso y, en la Figura II, los resultados obtenidos por todos los grupos en esta fase.

\section{Cuadro 2}

\section{Resumen de estudiantes del Grupo I}

\section{El depósito}

Es el contrato por el cual una persona (depositario) se obliga para con otra (depositante) que le ha confiado una cosa mobiliaria corporal, a conservarle y a restituirle cuando le sea requerida. La finalidad del depósito es la conservación que se le impone a la parte que recibe la cosa.

Cuando el depósito es gratuito, este solo origina obligaciones con cargo al depositario, dado que el depositante no tiene obligación de entregar la cosa; sin embargo, cuando el depósito es retribuido, la retribución misma se convierte en la obligación del depositante.

En este sentido, las obligaciones del depositario son la conservación y la restitución de la cosa cuando se extinga el contrato y las del depositante son, además de pagos la remuneración convenida, la de reembolsar los gastos hechos por el depositario y reparar los prejuicios que le haya causa la cosa.

Como medio de asegurar que el depositante cumpla, el depositario se beneficia de la garantía del derecho de retención, estipulado en el artículo 1948 código civil.

En el anterior resumen se aprecian avances con relación a la primera fase del proyecto, lo que denota que los estudiantes van comprendiendo que deben omitir, seleccionar y jerarquizar ideas para realizar un resumen. En efecto, este texto es más corto que el realizado en la fase diagnóstica, posee mayor cohesión y se han eliminado las listas de ideas. Sin embargo, aún se mantiene la literalidad con respecto al texto base, lo que demuestra la necesidad de continuar trabajando procesualmente en clase para lograr mejorar esta competencia. 
Figura II

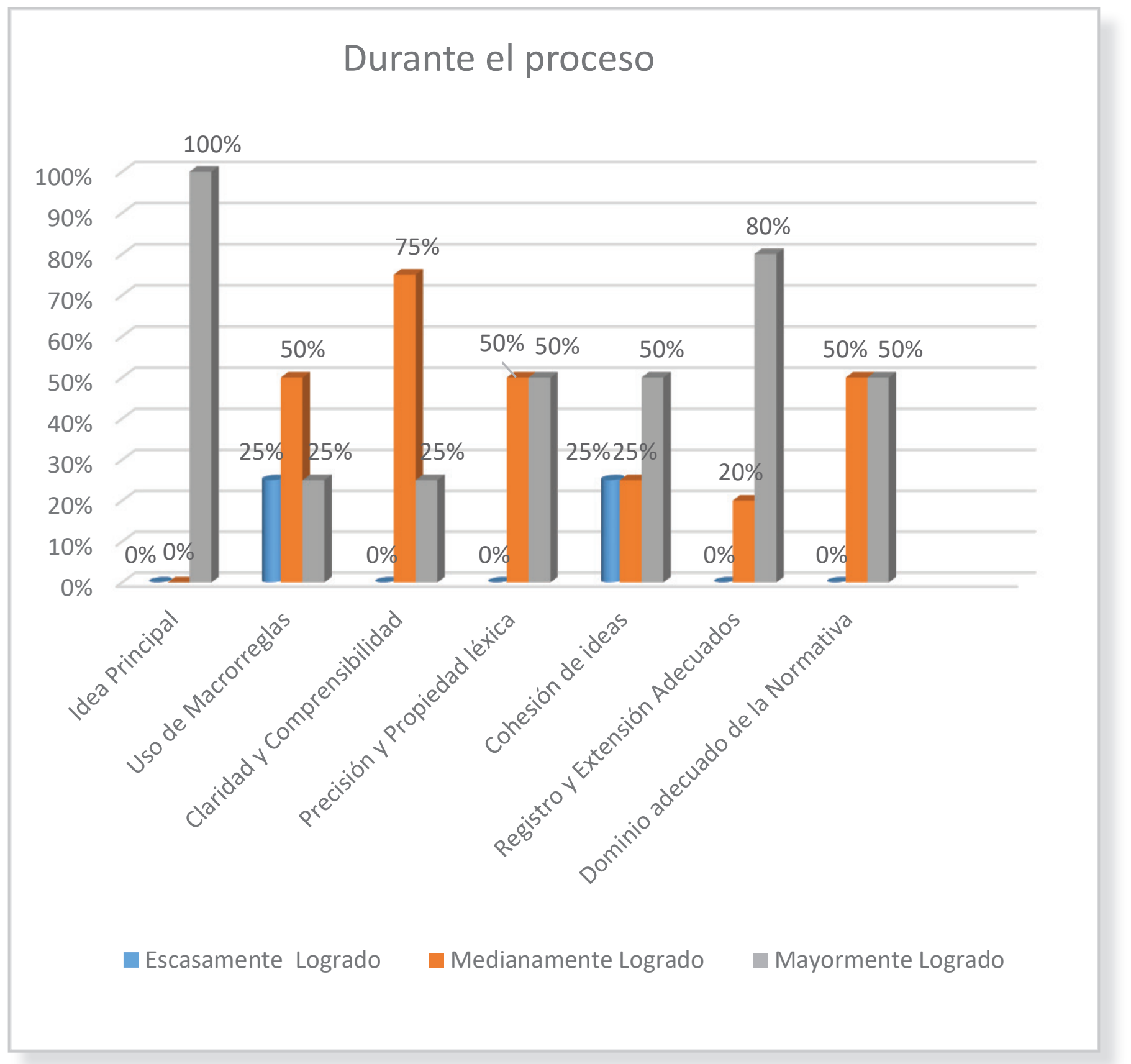

Se puede observar que, en esta fase, los alumnos escribieron los resúmenes presentando las ideas principales, mostrando ya la supresión de la técnica del listado y aplicando las tres primeras macrorreglas: omisión, selección y generalización, aunque aún falta un camino por recorrer para que cohesionen mejor sus ideas y dominen las macrorreglas de forma más competente.

Veamos ahora la cuarta y última fase del trabajo, en la que se incluyó un escrito para ser resumido en el examen final y de manera individual.
En este caso, se decidió que esta tarea se haría individualmente como culminación del proceso de trabajo realizado durante todo el período.

Por asuntos de espacio y, a modo de ejemplo, a continuación, se transcribe un resumen de los realizados por los estudiantes durante la cuarta fase del proyecto de investigación. Luego se muestra la Figura N. 3, en la que se sintetizan los resultados de los resúmenes realizados por todos los estudiantes en esta última fase del proyecto. 


\section{Cuadro 3}

\section{Resumen de un Estudiante}

\section{Contrato de Transacción}

El contrato de transacción, contemplado desde el artículo 2044 al 2058 del Código Civil de la República Dominicana, es un convenio mediante el cual las partes evitan o ponen fin a una controversia haciéndose concesiones recíprocas. El mismo se caracteriza por ser sinalagmático, a título oneroso, conmutativo y consensual. Dicho contrato produce diversos efectos, entre los que se puede señalar que es declarativo y que no obliga a terceras personas.

El anterior resumen es coherente y tiene una extensión adecuada. Posee conectores y muestra las ideas principales; se utilizaron eficazmente las macrorreglas, con excepción de la construcción del nuevo texto, que es un aspecto que aún puede mejorarse.

\section{Figura III}

\section{Evaluiación final}

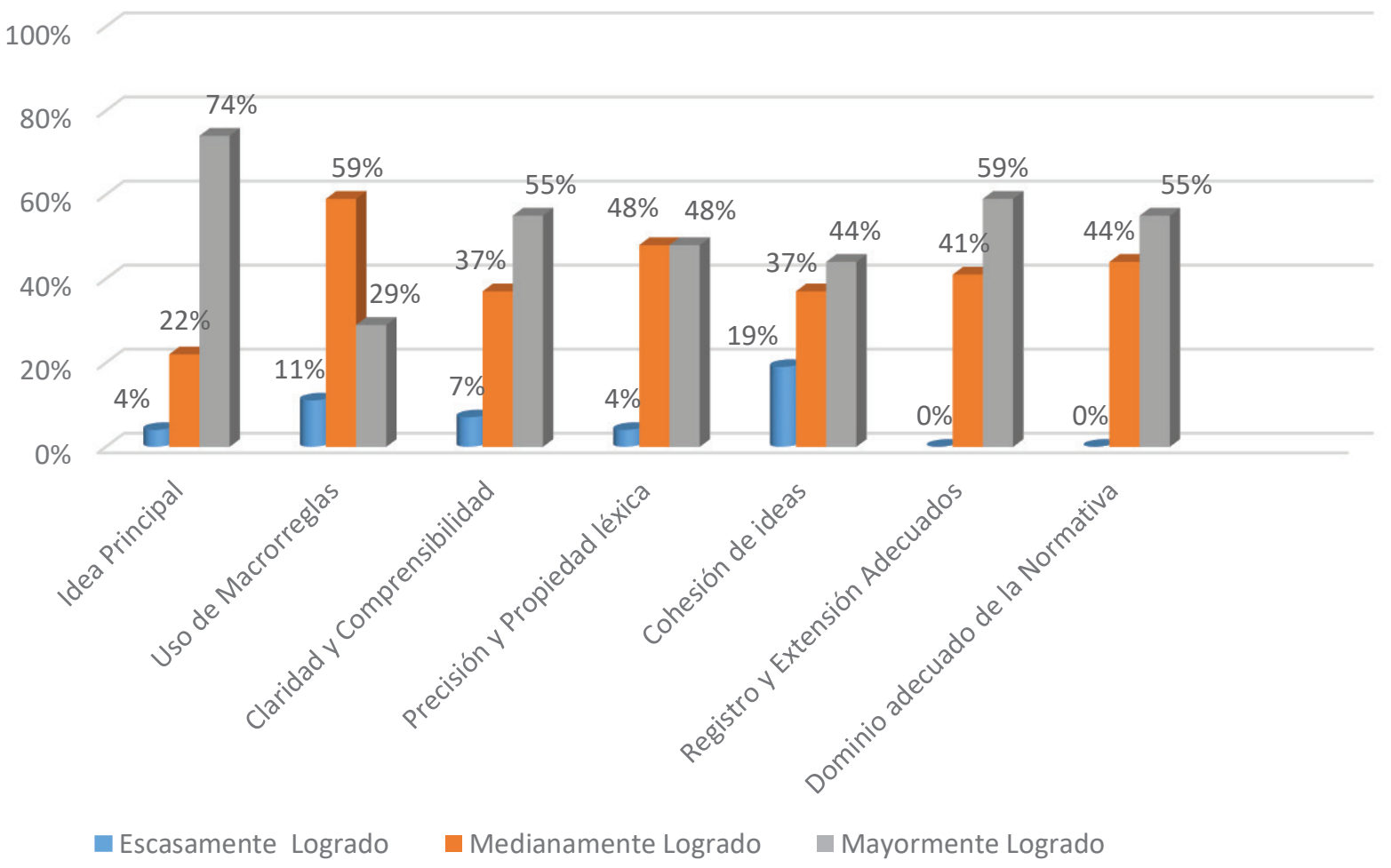

Como puede apreciarse en la Figura III, en los resúmenes finales existe notable mejoría en el manejo de esta estrategia por parte de los estudiantes. Hay grandes avances en la presentación de la idea principal e ideas que la apoyan. La aplicación de las macrorreglas de omisión y selección están muy bien logradas, al igual que la de la generalización, evidenciando un eje organizador de la información. 
Finalmente, se aprecia un buen dominio de la normativa. Falta por perfeccionar la aplicación de la regla de construcción, pues aún se mantiene literalidad del nuevo texto respecto del texto base, así como mejorar la cohesión entre las ideas, ya que algunos estudiantes no usan aún conectores apropiados y esto atenta contra la progresión lógica de las ideas.

\section{Conclusión}

Luego de haber implementado la estrategia del resumen para mejorar la compresión de los textos entre los estudiantes del área jurídica, se puede afirmar que los resultados han sido satisfactorios. En cada una de las cuatro fases de este proyecto de investigación en la acción, resultó notorio que los estudiantes mostraron avances en la elaboración de los resúmenes, lo que potenció su conocimiento disciplinar.

Se pudo percibir, en cada una de dichas fases, la progresiva identificación de los estudiantes participantes con los propósitos del proyecto. Su interés fue más allá de simplemente cumplir con un requisito, pues valoraron que la estrategia del resumen les ayudó a mejorar la compresión de los textos que debían estudiar en la asignatura.

Se pudo apreciar que la realización de resúmenes como instrumento para mejorar la comprensión lectora constituye una estrategia que puede ser mejorada en breve tiempo, cuando va de la mano de una didáctica constructiva. En efecto, la intervención pedagógica que se realizó durante un semestre académico fue de gran ayuda para que los estudiantes de la asignatura Derecho de los Contratos mejoraran en la competencia del resumen.

Ahora bien, si los participantes avanzaron en el desarrollo de esta competencia en un sólo ciclo académico, pensamos que, si se incluye a lo largo de la carrera, se podrá profundizar más en la misma, sobre todo en lo que concierne al uso de las macrorreglas y de los conectores, con los que se lograría una mejor progresión lógica de las ideas, competencia esencial para un profesional del Derecho.

Para nosotros, como profesores, ha sido muy interesante comprender que el proceso de enseñanzaaprendizaje no debe de limitarse sólo al contenido disciplinar, ya que la implementación de estrategias de comprensión lectora, situadas en el área jurídica, permitió potenciar el aprendizaje de los estudiantes.

Aplicar el resumen como estrategia para mejorar la comprensión lectora en la asignatura Derecho de los Contratos posibilitó que los estudiantes comprendieran la importancia del procesamiento de la información escrita en la comunidad científica a la que aspiran a pertenecer y que aprender una asignatura tiene que ver con el dominio de los recursos discursivos de dicha asignatura.

\section{Referencias}

Barrera, A. \& Zamudio L. (2011). La historia de la compraventa. Andres barrera.wordpress.com

Carlino, P. (2013). Alfabetización académica diez años después. Revista Mexicana de Investigación Educativa. México: Consejo Mexicano de Investigación Educativa, 57, 355-381.

Castelló, M. (2008). From self-o to socially regulation of writing: Different voices in graduate students 'writing.International Journal of Psychology, 43 (23), 356-366.

Castillo, A., Santiago (2005). Formación Del Profesorado En Educación Superior. D Hill/Interamericana de España, S. A. U.

Código Civil República Dominicana. Legislación Complementaria. (2013) República Dominicana. Moca: Editora Dalis.

Padilla, C., Douglas, S. \& López, E. (2010). Yo expongo. Taller de prácticas de comprensión y producción de textos expositivos. Córdoba: Comunicarte.

Padilla, C., Douglas, S. \& López, E. (2011). Yo argumento. Taller de prácticas de comprensión y producción de textos argumentativos. Córdoba: Comunicarte.

Romero B. (2001). Tratado Elemental De Derecho Civil. República Dominicana: Ediciones Jurídicas Trajano Potentini.

Van Dijk, T. (1980). Macrostructures. An Interdisciplinary Study of Global Structures in Discourse, Cognition and Interaction. Hillsdale (Nueva 